This item was submitted to Loughborough's Research Repository by the author.

Items in Figshare are protected by copyright, with all rights reserved, unless otherwise indicated.

\title{
Measuring the risk of sustaining injury in sport a novel approach to aid the re-design of personal protective equipment
}

PLEASE CITE THE PUBLISHED VERSION

http://dx.doi.org/10.1016/j.apergo.2011.12.010

PUBLISHER

(c) Elsevier Ltd and The Ergonomics Society

VERSION

AM (Accepted Manuscript)

LICENCE

CC BY-NC-ND 4.0

\section{REPOSITORY RECORD}

Velani, Nikunj, Ollie Wilson, Ben J. Halkon, and Andy R. Harland. 2019. "Measuring the Risk of Sustaining Injury in Sport a Novel Approach to Aid the Re-design of Personal Protective Equipment". figshare. https://hdl.handle.net/2134/13139. 
This item was submitted to Loughborough's Institutional Repository (https://dspace.lboro.ac.uk/) by the author and is made available under the following Creative Commons Licence conditions.

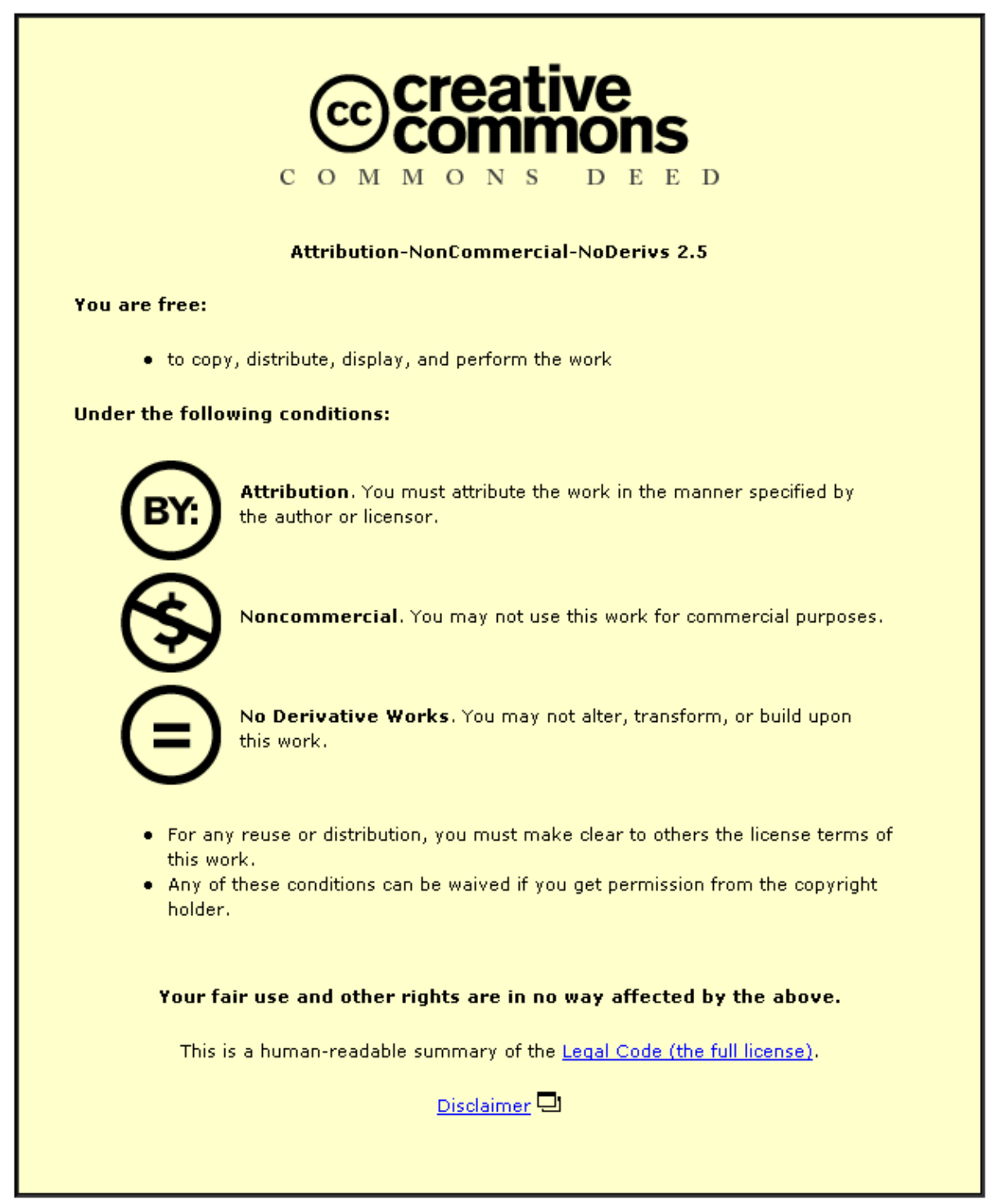

For the full text of this licence, please go to: http://creativecommons.org/licenses/by-nc-nd/2.5/ 


\title{
Measuring the Risk of Sustaining Injury - A Novel Approach
}

\author{
Nikunj Velani, Ollie Wilson, Ben J. Halkon, Andy R. Harland \\ Sports Technology Institute, Loughborough University, 1 Oakwood Drive, Loughborough, \\ Leicestershire LE11 3TU, UK
}

\section{Abstract}

Despite the possibilities offered by new approaches in design and advances in materials and manufacturing methods, few items of Personal Protective Equipment (PPE) used in sport have seen significant change for many decades. A major reason for this is the tradition and conservative attitudes associated with many sports, although the absence of appropriate tools and techniques to assist the design and development process has also played a large part. The aim of this study was to develop the first stage of a method of identifying specific regions of the human anatomy that are at the greatest risk of sustaining injury during participation in sports in which the player is subjected to multiple ballistic impacts. It is proposed that the findings could be used to confirm future designs of sports PPE. Previous studies have identified the amount and the location of the protection provided by current commercially available products but, until now, no evidence has been reported to determine what protection is required based on an understanding of the likely impact and the anatomy of the athlete.

Using the leg and cricket as examples of an anatomical feature and a sporting application respectively, the severity and probability of injury due to ball impacts typically observed in play are quantified, with respect to their location on the leg, to estimate the level of risk in that region. Results show that the level of risk is greatest in the shin regions of the front leg, suggesting that this region should be offered the greatest degree of protection, as is generally the case in commercially available leg guard designs. Conversely, however, the inner region of the mid-shin of the back leg is at the lowest risk, suggesting that protection in this region might be substantially reduced, a feature which is certainly not included in current product; such a reduction may significantly enhance the ergonomic performance of the leg guard design.

The findings of this preliminary study indicate that the method offers the potential to quantify the relative risk of sustaining injury, in a sports specific application, as a function of location on the body and is thus a potentially useful design tool for design engineers of sports PPE. Given the embryonic nature of this approach, however, a number of assumptions and additional considerations are presented which reveal that, whilst the technique offers additional design insight, further research is required before it should be applied to equipment design. 


\section{Introduction}

Participation in virtually all sport carries the risk of sustaining injury through impact, either from a bat, ball, club or another participant. Some sports, such as hockey and football, carry a significant risk of injury and, as a result, the laws of the games stipulate that Personal Protective Equipment (PPE) must be worn (e.g. shin guards in football [FIFA, 2010] and goalkeepers hand and leg guards in hockey [FIH, 2011]). Athletes, however, often feel the need to use additional PPE to further protect vulnerable areas despite the laws not enforce their usage. In some cases, such as in cricket, no laws exist in the senior game that enforce players to wear protective equipment yet countless PPE articles are still worn. Where it is discretionary, an athlete will make a choice as to which items of PPE are worn based on a compromise between their personal safety (the protection afforded by the PPE), comfort (during and after wear) and performance (accounting for any detriment in performance caused by wearing PPE).

In order to be sold in the UK, all PPE items must pass specific test standards to certify that they are of an adequate protective standard (McIntosh et al., 2003), for example the BS 6183-3:2000. The majority of these standards have been developed focusing solely on the safety aspects. It has therefore been very difficult for a designer or manufacturer to improve the comfort or performance aspects of PPE without compromising safety, such as is the case for the cricket leg guard, used as an exemplar in this study, where no single injury incident has been reported in over a century of use. In contrast, there are no standards specifically defining design requirements of PPE (McCrory, 2002), with reference to design only being made to state that the sports PPE must allow typical athlete movements (e.g. BS 6183-3:2000, BS EN 13061:2009). As a result, the design is often neglected in the development process and modern sports PPE can be found to exhibit satisfactory protective capabilities but be ill-fitting, bulky and/or heavy, ultimately inhibiting the performance of the athlete using it (Akbar-Khanzadeh et al., 1995; Webster and Roberts, 2009). In some cases, this has led to athletes modifying their own PPE in an attempt to make it more comfortable to wear or less detrimental to performance with little consideration on how it may affect the safety characteristics of the PPE. The lack of specifications constraining designs enables the possibility of re-invention that could counter the problems observed in existing PPE yet, despite this, few variations from the norm are found. Striving to minimise athlete inhibition (thus maximise performance potential), in today's games, is of particular importance where success and failure are often separated by the smallest of margins.

There is no one clear definition for 'injury' but the categorisation of injury in sport is most commonly performed by using two main classes: 'medical attention injuries' (i.e. those which require treatment 
by a medical practitioner) and 'time-loss injuries' (i.e. those which cause athletes to miss one or more practice or game sessions) (Bahr and Engebretsen, 2009). Injuries that require the greatest medical attention or the greatest time lost are those that are deemed to be the most severe and, ultimately, those that are the most undesirable. Of all injuries sustainable from impact in sports, fractures can be argued to carry the greatest severity and have been reported to cause up to 240 playing days lost during recovery time (Oztekin et al., 2009). They occur when the kinetic energy of the impactor that is translated to the body exceeds the fracture energy of the bone (Ankrah and Mills, 2003). Clearly the fracture energy is most likely to be exceeded when the impact energy has the least opportunity to be dissipated (i.e. where bones are superficial to the skin). Bones that are deep are typically protected by soft tissue, which can absorb sufficient energy to ensure that the energy translated to the bone is less likely to cause fracture. Impact with regions that have deep lying bone, therefore, most commonly result in an injuries of lower severity, usually contusions which, in the worst case, have been reported to cause only 60 playing days loss (Oztekin et al., 2009).

PPE has been observed to reduce the injury rate in a range of sports but ultimately not eliminate injuries completely (Marshall et al., 2002; Mclntosh and McCrory, 2005; Benson et al., 2009). Furthermore, these injuries have often been reported to be focused in particular anatomical regions (Leary and White, 2000; Marshall et al., 2002; Finch, 2002; Stretch, 2003) suggesting that, in general, some parts may be over protected and others under protected. Injuries sustained during batting in cricket provide a prime example of this theory. Milsom et al., (2007) reported that $10 \%$ of all injuries during batting were phalangeal fractures, whilst there were no reported incidences of injuries caused in the lower limbs. Given that both regions are protected by PPE, it can be argued that some protection in the leg can potentially be reduced without increasing injury occurrence, but that protection in the fingers must be increased to reduce the number of injuries sustained. The challenge in achieving this, however, lies with the determination of where and the extent to which protection can be removed, or increased, in order to develop PPE that is injury risk minimising, minimally performance inhibiting and ergonomic in design. To arrive at this balance it may be acceptable, for example, to develop PPE that shows an increased level of low severity injuries, such as contusions which do not prevent the athlete from playing if sustained, if the newer design displays better ergonomic and performance characteristics than its predecessors. The lack of required information, with respect to the areas in which the protection is most necessary and the sustainable injury levels that are acceptable, combined with the associated inertia to move away from existing designs, has arguably not allowed modern technological and material advances to be fully incorporated into PPE designs to make such changes possible. 
Areas that are the most susceptible to sustaining a severe injury are those that theoretically require the greatest protection from PPE. Areas that may be likely to sustain severe injury, however, may not require any protection should the region have little chance of sustaining an impact. This relationship between probability and severity and its influence on sustaining injury has often been defined as a measure of the 'risk of injury' (Anthony Cox, 2008; Bahr and Engebretsen, 2009; BS OHSAS 18001:2007). Risk estimation has most commonly been conducted using three quantitative techniques: 1) Risk matrix (estimation of risk by considering the consequence [or severity] and probability of injury), 2) Fine and Kinney's method (estimation of risk based on the consequence, exposure rate and the probability) and 3) Quantitative risk measures of societal risk (estimation of risk based on the accident category, frequency and consequence) (Marhavilas and Koulouriotis, 2008). Of the three approaches the risk matrix approach presents the simplest, and broadest, method of estimation suggesting why it has been used in the HSE (2010) and widely employed in studies, such as those by Drawer and Fuller (2002) and Greenwald et al., (2008), to estimate the risk of sustaining an injury.

Within the sporting domain, risk has been estimated as a function of the perception of occurrence (Rundmo, 1996) or, more commonly, the frequency of injury over an extended period of time (Woods, 1996; Leary and White, 2000; Finch, 2002; Stretch, 2003; Marshall et al., 2005; Mclntosh and McCrory, 2005). Little work, however, has been conducted on analysing the risk as a function of the human anatomy. Woods (1996) successfully presented a method of determining what type of protection was required in certain regions based on the occurrence rates of the types of damage that prevailed on motorcycle suits after accidents. Although the method allowed modern motorcycle protective suits to be tailored to prevent these forms of damage, hence minimising the risk of injury at that anatomical location, the study also suggested that some of the injuries were sustained irrespective of whether that additional protection was present or not. The method presented, therefore, may not necessarily be the optimal means to analyse sports PPE to aid designers in making protective modifications, especially where most sporting impacts cause little visible damage to PPE. More recent studies have focused on the analysis of the capabilities of current protection based on the type of injury that could be sustained (Marshall et al., 2005; Milsom et al., 2007), but have eluded to state how protection could be adapted to alter the risk. They have not, therefore, provided designers with the information that is required to make modifications to counter the drawbacks of current sports PPE.

This study aims to present a method in order to identify where the greatest relative risks of sustaining injury lie across a region using the risk matrix method for a single sample right-handed 
batsman, with the leg and cricket leg guard as examples of a potential anatomical feature and sporting application respectively. Understanding where the greatest risks lie and how they vary could form a useful design tool that could be implemented into the design process to aid designers in understanding where protection could be reduced or increased to optimise PPE design. By its nature, the method is specific to the user, application and the nature of the injury deemed most severe.

\section{Method Development}

The risk of sustaining an injury has previously been described as a product of the potential severity of injury and the probability of impact in a certain region (Equation 1) (Anthony Cox, 2008; Bahr and Engebretsen, 2009; BS OHSAS 18001:2007). If either the probability of impact or the severity of injury is low at a location the risk of sustaining an injury at that location is low. Similarly, if both are high the risk is high. Understanding this balance between the potential severity of injury and the probability of impact is of great importance when attempting to determine where and what degree of protection is needed.

$$
\text { Risk }=W_{\text {sev }} \cdot \operatorname{Sev} \times W_{\text {prob }} \cdot \text { Prob }
$$

Where: $\mathrm{W}_{\text {sev }}=$ Severity Weighting Function

$$
\mathrm{W}_{\text {prob }}=\text { Probability Weighting Function }
$$

In accordance to Equation 1, both the severity and probability are required to be critically weighted to arrive at a precise quantified injury risk value for a specific location but, due to the infancy of this approach and the consequent lack of available data to justify different weightings, this study utilises an equal weighting.

\subsection{Source Data Collection}

Forty-one magnetic resonance imagery 'slices' of a person's right leg, taken at $21 \mathrm{~mm}$ intervals between the ankle and the hip, were obtained for analysis from the Visible Human Server data set (EPFL, 2000). Each 'slice' was extracted from the data set as a 400x400 pixel JPEG image from which both hard tissues (bone) and softer tissues (skin, fat and muscle) could easily be identified.

\subsection{Potential Severity of Injury Estimation}

With the front of the leg facing upwards, the centre point of each 'slice' was found by locating the intersection of the $\mathrm{x}$ and $\mathrm{y}$ bisector of the maximum and minimum horizontal and vertical pixels, as 
shown in Figure 1a. This enabled 24 equi-spaced positions $\left(15^{\circ}\right.$ intervals) to be marked around the perimeter of the leg, as shown in Figure 1b. From each of these positions the shortest distance to a bone was measured and recorded, as can be seen graphically in Figure 1c. A smaller 'distance' represents an area that has a high potential severity of injury and vice versa.

The 'distances' for all slices were collated and normalised by subtracting the minimum value (from the entire collation [2]) from each individual value and then dividing the resultant by the range of values (88). Consequently a value close to 1 represented a lower severity of injury. The scale, however, was reversed, by subtracting each value from 1 , to arrive at data that was intuitively easier to interpret (higher values represent a higher severity). New values were then plotted on a single 'grey-scale' map, as shown in Figure 2, such that the variance in the potential severity of injury could be observed both graphically and numerically as a function of the location on the leg. The darkest regions are indicative of areas that are considered susceptible to injuries of high severity, whilst lightest regions represent those least likely to sustain severe injury. During this analysis it was found that, at the transition between the leg and adjacent anatomical features, determining the perimeter of the leg from the image became increasingly difficult. To maintain the validity in the results slices 1 (lower leg/foot transition) and 39-41 (upper leg/hip transition) were eliminated from analysis for this reason.

The results presented in Figure 2 reveal that the potential severity of injury is greatest in the central regions on the leg in the mid to lower areas. Simple analysis of the slice numbers enables the determination of these areas to represent the knee and shin regions. Measurements between slices 20 and 24 (approximately the knee region) reveal a wide region in which the potential severity of injury is high, suggesting that protection requirements are greatest within the area extending from the medial to the lateral through a range of $180^{\circ}$ centred around the patella. The shin and calf region show a large range of measured distances. Whilst the presence of the tibia proximal to the skin at the front of the leg reveals areas of high injury risk; the large amount of soft tissue at the back of the leg is influential in reducing the risk rating.

\subsection{Probability of Impact Estimation}

In order to continue the analysis, an estimation of the likelihood of an impact occurring at a specific position is required. The leg was therefore divided into fifteen regions of interest, as shown in Figure 3. 15 shots (individual methods used to facilitate bat-ball impacts for different types of ball deliveries) typically played during batting were selected for analysis, for which images would be required. Ten of these shots were selected from Palmer (1999): the forward defence, front foot off/on drive, back foot defence, back foot off/on drive, the sweep, front/back foot leg glance and the 
leave. A further five: the reverse sweep, leg side 'slog', 'padding up' to a ball on the off/leg side and Yorker, were added to broaden the scope and better reflect shots played in contemporary professional cricket.

Images of the first ten shots being played by a right-handed batsman were obtained from Palmer (1999). The remaining five, which were not depicted in Palmer (1999), were captured from an alternative right-handed batsman, of the same experience, at or close to the point of ball impact. From these images, regions that were determined to be the most likely to sustain impact were given a score of 2, regions that were likely to sustain impact due to unexpected bounce or ball movement were given a score of 1 , whilst areas that had little or no likelihood of being impacted were given a score of 0 . Regions were assigned scores based on the location of the 'middle' (optimal position on bat for ball impact) of the bat prior to ball strike (as were shown in images). Regions closest to the 'middle' of the bat were assumed to have a greater probability of impact (assigned scores of 2 ) over those further away from the 'middle' of the bat (given scores of 1 or 0 ). Both the front and rear legs were taken into consideration given the inherent leg positions necessary to play all the shots involve both legs being exposed to impact by varying degrees. Unlike the case of the potential severity, the probability of impact is not symmetrical hence the necessity of both legs being considered when assigning values to various regions. For simplicity, this initial work excluded potential changes in possible impacted regions due to individual batsmen style, pitch and environmental conditions, etc. in determining whether the process was a viable method to estimate the risk of sustaining an injury. Impact probability ratings were collated for all 15 shots, normalised (using the process described in section 2.2) and presented in a single probability 'grey-scale' map, as shown in Figure 4. This enables the areas of greatest probability of impact to be readily viewed as a function of both the location on the leg and the leg of interest (front or rear). Darker areas and larger values on the map indicate regions that have the greatest probability of impact and, conversely, lighter regions and lower values indicate those least likely to sustain impact.

The combined data unsurprisingly, but reassuringly, show the front leg to have a substantially greater probability of impact than the rear leg thus indicating that the front leg should be provided with greater protection than the rear. The highest probabilities are found to be concentrated in the central and inner regions of the knee and shin regions of the front leg. Conversely, the inner mid region of the rear leg shows a zero probability of impact suggesting that minimal protection is required in this region. 


\subsection{Risk of Injury Determination}

Quantification of the potential severity of injury and the probability of impact enabled the risk of sustaining impact to be estimated based on the risk matrix relationship given in Equation 1.

Prior to estimating the risk, however, the fifteen regions defined in the probability of impact had to be mirrored onto the potential severity on injury 'grey-scale' map, such that the relevant regions corresponded. Key anatomical landmarks were used in order to determine the range of slices that represented the heights of the probability regions. For example, the thigh region was determined by the identifying where the slices last showed features solely found in the upper leg (i.e. only the femur observed), and subsequently the knee region from where initial knee features were observed to the slice prior to where they were no longer seen (i.e. only tibia observed). Slices that represented the remaining upper shin, mid shin and lower shin regions were determined by making calculated estimations based on the number of slices that represented the heights of the thigh and knee regions. Thus, the thigh was defined as slices 24-38, the knee: 20-23, upper shin: 14-19, mid shin: 813 and lower shin: $2-7$.

Lateral coverage was established such that the inner, central and outer regions were covered by an equal number of equi-spaced positions. The inner leg was consequently defined by positions A-H, the central leg by positions I-P and the outer leg by positions Q-X. This is shown for the front leg for a right-handed batsman in figure 5.

Risk was finally estimated, according to Equation 1, by multiplying the potential severity values by the probability of impact values of their respective regions, as displayed in Figure 5. Calculated values are presented graphically and numerically on a 'grey-scale' map for both the front and rear legs of a right-handed batsman, as shown in Figures 6 and 7, where darkest regions in the maps represent areas of high risk and the lighter regions areas of low risk.

Figure 5a displays the segregated regions of a single leg and the corresponding normalised probability values for both the front and rear leg (Figures $5 b$ and $5 c$ ) of a right-handed batsman. In the case of a left-handed batsman, however, to estimate the risk of sustaining an injury, values for the front and rear legs, for the probability of impact, would be required to be interchanged due to the change in orientation of batsman stance. So, for example, the inner mid shin region of the rear leg for a right-handed batsman is equivalent to the inner mid shin region of the front leg for a lefthanded batsman and thus should be assigned a value of 0.71 . Potential severity of injury values would remain consistent between right and left-handed batsmen because anatomically players would be alike. 


\section{Discussion}

\subsection{Validity of Approach}

This primary aim of this study was to develop a novel conceptual method of quantifying the risk of sustaining injury that participants face during a sporting discipline - in this case batting in cricket. The motivation for this approach was to provide equipment designers with data that might be used within the design process in order to create equipment that better fulfils the needs of the end user. Previous studies have yielded little information regarding the risk of injury as a function of anatomical features, as they have in the main focused on the locations of the injuries. This has provided designers with little useful information with regards to how modifications might be made to manage risk and safeguard against serious injury whilst ensuring the PPE minimally inhibits performance and is ergonomic in design. It is anticipated that the methodology proposed in this study overcomes this shortcoming and could, in theory, be applied to any part of the anatomy, for any sporting application, provided that appropriate input data could be sourced.

The findings of this study have suggested there to be a substantially greater risk of sustaining an injury during batting in cricket in the front leg than in the rear leg, with the greatest risk present within the central mid shin region of the front leg. Regions of high risk were found to be concentrated in the upper and mid shin regions of the front leg and the lower thigh and knee regions of the rear leg, detailing where the protection might be concentrated on each. Given the proximity of the bone to the skin and the leg positions required to play the fifteen shots analysed, this finding is likely to align with any experienced athletes perceptions, and adds justification to the British Standard (BS 6183-3:2000, 2010), which requires additional protection within the shin regions. Individual measurements have shown that both the potential severity and the probability of impact were found to be greatest in the central shin region, thereby leading to the greatest risk being found in this region, correct with previous reported findings. The same region on the back leg, however, was shown to be exposed to a considerably lower risk, suggesting that the observed symmetrical nature of the front and rear leg protection afforded within commercially available leg guards may not necessarily be justified. The lowest risk of injury was found to be in the inner mid shin region of the rear leg which was estimated to be completely free from risk due to the zero probability of impact in this region for the range of shots studied. This suggests that it may be possible to substantially reduce the amount of protection, without substantially increasing the risk of injury for the batsman.

Analysis of the risk in this manner would enable PPE designers to visualise the relative protection needs of different regions of the protective equipment. Whilst the calculated values do not indicate 
the absolute amount and exact type of protection that should be afforded in novel designs, they do, however, provide invaluable information with respect to manufacturing a 'functionally graded' PPE (i.e. one that exhibits different protection properties in different areas). For the purpose of converting the values into design elements, understanding all the mechanisms (peak force, pressure, strain, etc.) that lead to severe injury within the body and the complete array of design elements (stiffness, strain rate, fracture toughness, mass, density etc.) that lead to a design being able to mitigate the risk of sustaining injury more effectively than previous versions, is fundamental. By this mechanism, the method would become an effective tool for the redesign of sports PPE.

In accordance to the presented analysis, where the estimated risk of injury is 1 the greatest amount of protection is required and where the estimated risk is 0 protection can theoretically be alleviated on that particular article of PPE. As a result, scales ranging from maximum to minimum could be developed to indicate the required quantity of a particular material, that is required to minimise the risk of severe injury occurring for a particular region.

The method also lends itself to the possible manufacture using multiple materials that display varying 'protective capabilities'. For example, in cricket leg guards, different amounts of a single material can be selected for various regions such that both protection and design element requirements are met. Furthermore, combinations of materials can be selected such that each material provides sufficient protection to individual regions, whilst also adhering to the required design element requirements of that particular region. This will further reduce the risk of a cumbersome solution being developed, the like of which may be observed in a single material design.

PPE should ideally minimise the inhibition to performance whilst keeping the athlete injury risk free. The developed method also potentially allows PPE to be tailored for individual athletes based on their i) specific batting technique or the range of shots that they most commonly play, ii) the general pitch condition, iii) where in the world the match is played, iv) the format of the game etc.,. One, or all, of these variables may result in significant differences in the area(s) of greatest injury risk.

\subsection{Limitations of the Study}

With the advent of shorter forms of the game, the range of shots being played is ever increasing with many batsmen adopting unconventional techniques in an attempt to score more runs more rapidly. In these cases, whilst the method of estimating the risk from an assessment of the probability and severity of impact remains a valid approach, the body positions assumed to play the fifteen identified shots would almost certainly not be appropriate. Accommodating match specific 
scenarios where athletes innovate new techniques and methods to gain a competitive advantage, or taking into account the frequency with which different shots are played or those deliveries which are not successfully played by the batsman, would require further analysis of individual approaches. A similar situation exists in the case of a left-handed batsman. The angle of ball delivery (and shot selection, as a result) may generate different regions of possible impact when compared to a righthanded counterpart. Consequently, the method should not be used to develop symmetrical sports PPE for left-handed players based on the findings of a right-handed player but, instead, serve as a tool to enable PPE to be tailored for this alternate, left-handed batting scenario. As a as a general tool for conventional match play, however, the approach presented is considered valid.

In order to quantify the severity of injury it was assumed that both legs were perfectly symmetric and that the severity varied linearly with the distance from the bone surface to the skin; bone that was deep was assumed to be at less risk than that which was superficial. Muscle, however, can exist in both relaxed and tense form, with the differences between them severely influencing impact energy absorption characteristics (Tsui and Pain, 2008). Muscle that is tensed has a significantly lower ability to absorb energy, thus translating a greater amount to the bone and potentially resulting in a greater potential severity of injury. Furthermore, the property of bone and its susceptibility to damage was considered to be homogenous, something that is unlikely to be the case due to both the structure of the skeletal arrangement and the localised bone material properties. Consequently, the method described should not be used as an indicator of likely damage for a specific impact, rather a generalised method of describing relative risk. Further research would also be required to allow the method to account for other causes of significant injury, such as contusions.

In accordance with Equation 1, both the severity and the probability values should include a weighting factor. In this study, however, the weightings for both the potential severity and the probability were kept at one because of a lack of previous studies or data supporting, and justifying, an alternately balanced weighting. The manner in which likelihood and severity are weighted is likely to be influenced by the aspirations of the athlete. Different athletes will place different value on staying free of injury, remaining comfortable whilst using PPE or optimising their performance. This may also change depending upon whether an athlete is playing a short, twenty over match or a longer match played over several days. Therefore, some athletes may be more tolerant to repeated impacts of low severity, whereas others may not. In accommodating this range of circumstances it is likely that the weightings will be adjusted. 


\section{Conclusions and Further Work}

Minimising the risk of injury is the primary design criterion for PPE. However, tools to identify where protection is most required and where it might be reduced such that users remain injury risk free but also minimally inhibited are sparse. The method reported in this study allows the risk of injury to be quantified in different anatomical regions based upon their potential severity of injury and probability of being impacted. In turn, this would allow designers to achieve a different balance between athlete safety, comfort and performance inhibition where required. The proposed method is suitable in scenarios where current PPE arguably offers excessive or inadequate amounts of protection. This study focussed mainly on the former situation but the method proposed would also allow designers to add protection where required.

In order to validate this method, continuation of the work should aim to use the data to manufacture a physical prototype leg guard, possibly using the approach suggesting in section 3.1, thus enabling both laboratory and player testing to be completed. A physical model would aid in the elimination of some of the limitations described in section 3.2 (and potentially reveal some that $s$ have so far been overlooked) in future iterations through direct input from both laboratory testing results and player feedback. Through the development and evaluation of a physical model, both the values and shortcomings of the risk assessment technique can be readily determined.

To maximise the value of the approach outlined, further research is also required to better characterise the damage mechanisms leading to playing days lost and the likelihood of particular impact types. Further research is also likely to allow specific applications or individuals to be considered based on bespoke input data and also consider scenarios where athletes may not necessarily be performing optimally or conventionally. Whilst this study only focussed on the leg and cricket leg guards the methodology is believed to be easily employable into all sports for any type of PPE to aid in the development of new designs providing the source data is available. 


\section{References}

BS 6183-3:2000. Protective equipment for cricketers. Leg protectors for batsmen, wicket-keepers and fielders, and thigh, arm and chest protectors for batsmen. 2010. London: British Standards Institution.

BS EN 13061:2009. Protective Clothing. Shin guards for association football players. Requirements and test methods. 2009. London: British Standards Institution.

BS OHSAS 18001:2007. Occupational health and safety management systems. Requirements. 2007. London: British Standards Institution.

AKBAR-KHANZADEH, F., BISESI, M.S. and RIVAS, R.D., 1995. Comfort of personal protective equipment. Applied Ergonomics, 26(3), pp. 195-198.

ANKRAH, S. and MILLS, N.J., 2003. Performance of football shin guards for direct stud impacts. Sports Engineering, 6(4), pp. 207-219.

ANTHONY (TONY) COX, L., 2008. What's Wrong with Risk Matrices? Risk Analysis, 28(2), pp. 497-512.

BAHR, R. and ENGEBRETSEN, L.R., Sports Injury Prevention (Olympic Handbook Of Sports Medicine) WileyBlackwell.

BENSON, B.W., HAMILTON, G.M., MEEUWISSE, W.H., MCCRORY, P. and DVORAK, J., 2009. Is protective equipment useful in preventing concussion? A systematic review of the literature. British journal of sports medicine, 43(Suppl 1), pp. i56-i67.

DRAWER, S. and FULLER, C.W., 2002. Evaluating the level of injury in English professional football using a risk based assessment process. British journal of sports medicine, 36(6), pp. 446-451.

EPFL, Visable Human Server. Available: http://visiblehuman.epfl.ch/ $[15 / 11,2010]$.

FÉDÉRATION INTERNATIONALE DE FOOTBALL ASSOCIATION (FIFA), Laws of the Game [Homepage of FIFA], [Online]. Available: http://www.fifa.com/mm/document/affederation/generic/81/42/36/lawsofthegame 201011 e.pdf [04/28, 2011].

FEDERATION OF INTERNATIONAL HOCKEY (FIH), Rules of Hockey [Homepage of FIH], [Online]. Available: http://fihlive.sportcentric.net/vsite/vfile/page/fileurl/0,11040,1181-203465-220688-168858-0-file,00.pdf [04/28, 2011].

FINCH, C., COSTA, A.D., STEVENSON, M., HAMER, P. and ELLIOTT, B., 2002. Sports injury experiences from the Western Australian sports injury cohort study. Australian and New Zealand Journal of Public Health, 26(5), pp. 462-467.

GREENWALD, R.M., GWIN, J.T. and CHU, J.J. AND CRISCO, J.J., 2008. Head impact severity measures for evaluating mild traumatic brain injury risk exposure. Neurosurgery, 62(4), pp. 789-798.

HEALTH AND SAFETY EXECUTIVE (HSE), Risk Based Approach - Risk Model (Initial Decision Matrix) [Homepage of HSE], [Online]. Available: http://www.hse.gov.uk/foi/internalops/og/ogprocedures/complaints/riskmodel.pdf [04/29, 2011].

LEARY, T. and WHITE, J.A., 2000. Acute injury incidence in professional county club cricket players (19851995). British journal of sports medicine, 34(2), pp. 145-147. 
MARHAVILAS, P.K. and KOULOURIOTIS, D.E., 2008. A risk-estimation methodological framework using quantitative assessment techniques and real accidents' data: Application in an aluminium extrusion industry. Journal of Loss Prevention in the Process Industries, 21(6), pp. 596-603.

MARSHALL, S.W., WALLER, A.E., DICK, R.W., PUGH, C.B., LOOMIS, D.P. and CHALMERS, D.J., 2002. An ecologic study of protective equipment and injury in two contact sports. International journal of epidemiology, 31(3), pp. 587-592.

MCCRORY, P., 2002. The role of helmets in skiing and snowboarding. British journal of sports medicine, 36(5), pp. 314-314.

MCINTOSH, A.S., MCCRORY, P., FINCH, C.F., CHALMERS, D.J. and BEST, J.P., 2003. Rugby headgear study. Journal of Science and Medicine in Sport, 6(3), pp. 355-358.

MCINTOSH, A.S. and MCCRORY, P., 2005. Preventing head and neck injury. British journal of sports medicine, 39(6), pp. 314-318.

MILSOM, N.M., BARNARD, J.G. and STRETCH, R.A., 2007. Seasonal incidence and nature of cricket injuries among elite South African schoolboy cricketers. South African Journal of Sorts Medicine, 19(3), pp. 80-84.

OZTEKIN, H.H., BOYA, H., OZCAN, O., ZEREN, B. and PINAR, P., 2009. Foot and ankle injuries and time lost from play in professional soccer players. The Foot, 19(1), pp. 22-28.

PALMER, G.V., 1999. Cricket Coachmasters Batting Mechanics. Gary Palmer.

RUNDMO, T., 1996. Associations between risk perception and safety. Safety Science, 24(3), pp. 197-209.

STRETCH, R.A., 2003. Cricket injuries: a longitudinal study of the nature of injuries to South African cricketers. British journal of sports medicine, 37(3), pp. 250-253.

TSUI, F. and PAIN, M.T.G., 2008. The effects of muscle tension on human biomechanical response and perceived impact intensity, Proceedings of the 4th North American Congress on Biomechanics, Ann Arbor, USA, August 2008.

WALKER, P.J., ROTHBERG, S.J., HOLMES, C.E. and HARLAND, A.R., 2010. Design of a force acquisition system for high-energy short-duration impacts. Proceedings of the Institution of Mechanical Engineers, Part P: Journal of Sports Engineering and Technology, 224(2), pp. 129-139.

WEBSTER, J. and ROBERTS, J., 2009. The Influence of Cricket Leg Guards on Running Times and Stride Parameters. , August 2009 2009, American Society of Biomechanics.

WOODS, R.I., 1996. Specification of Motorcyclists' Protective Clothing Designed to Reduce Road Surface Impact Injuries. ASTM SPECIAL TECHNICAL PUBLICATION, 1237, pp. 3-22. 

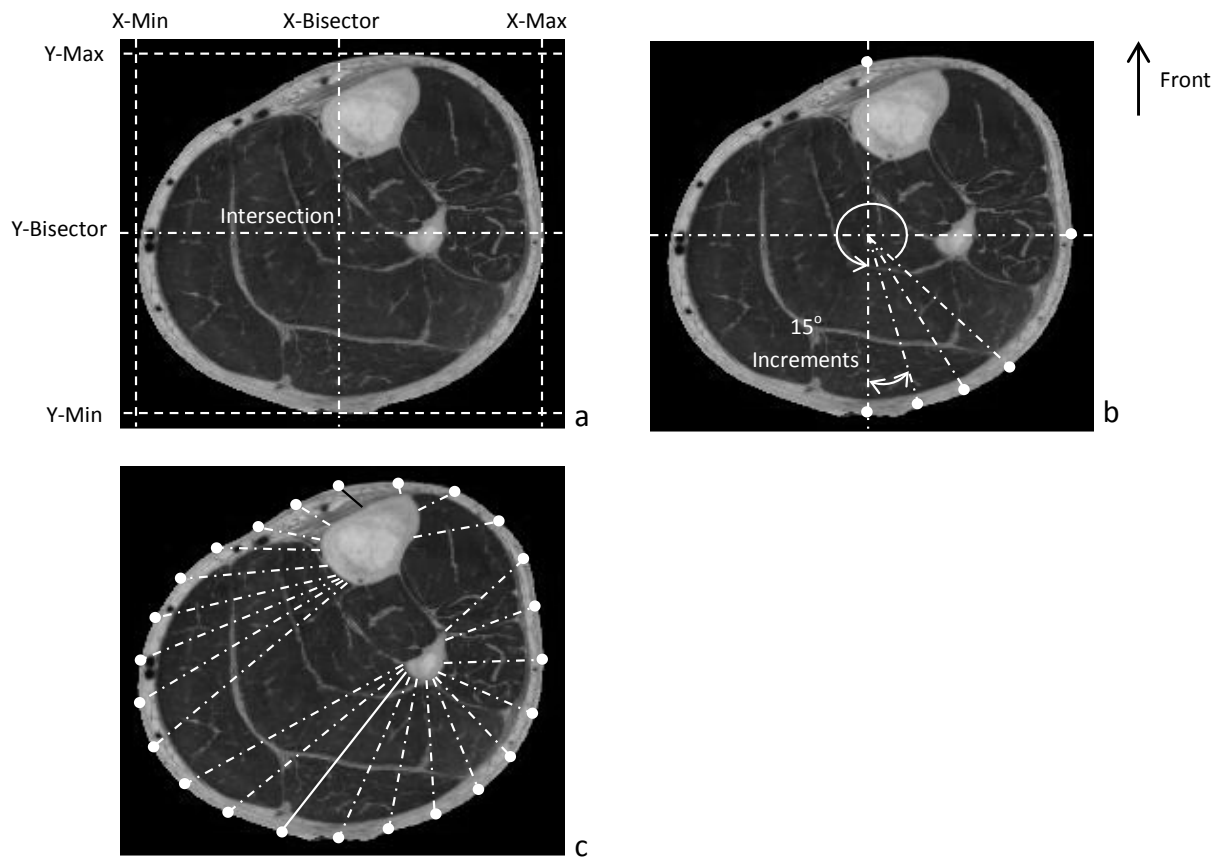

Figure 1. a) Calculation of the centre point of each slice. b) $15^{\circ}$ increment to obtain positions around perimeter of leg. c) Shortest distance to nearest bone (solid white line to position $X$ and solid black line to position $M$ ). 


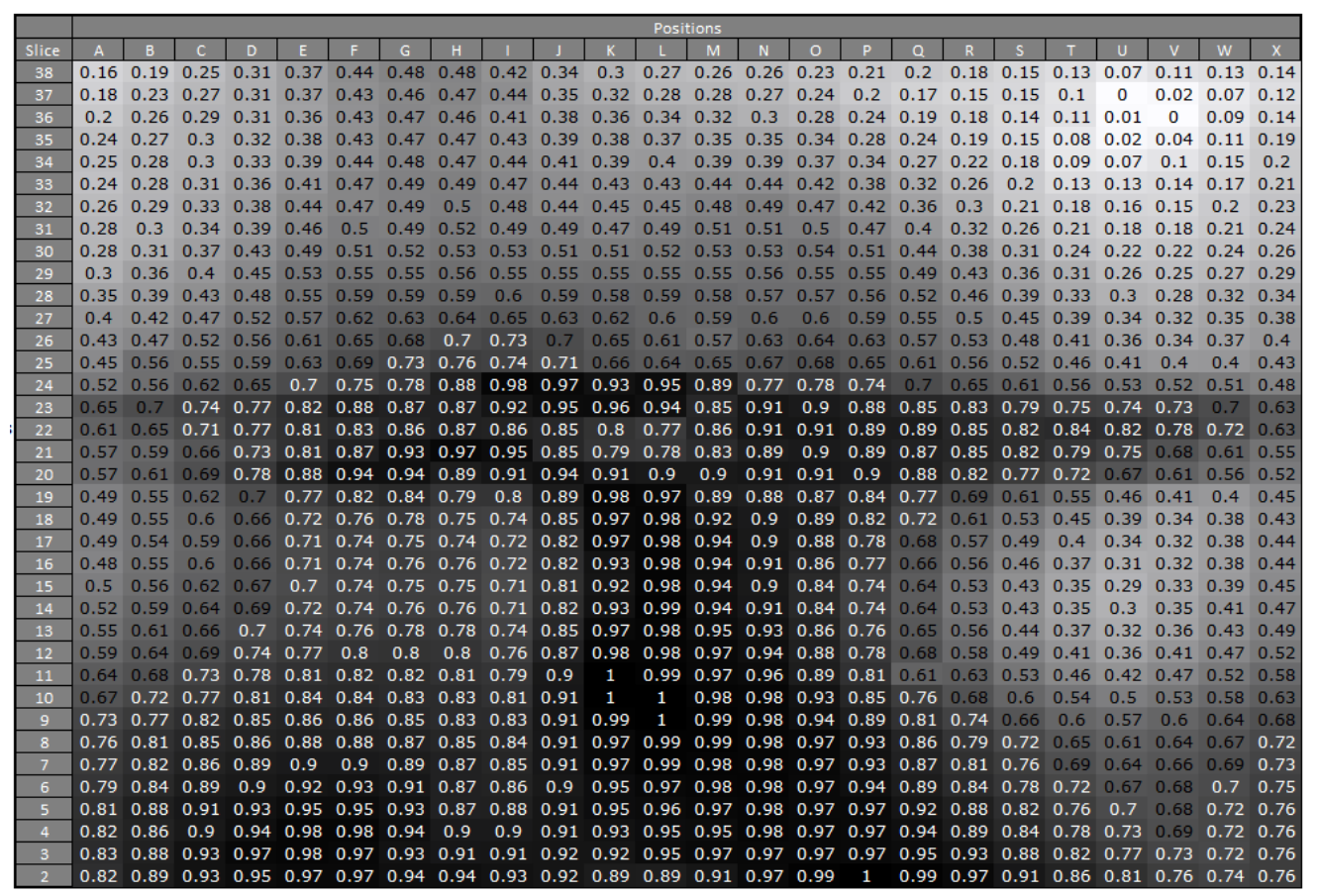

Figure 2. Normalised potential severity of injury 'grey-scale' map for a single leg with respect to 'height' on the leg 


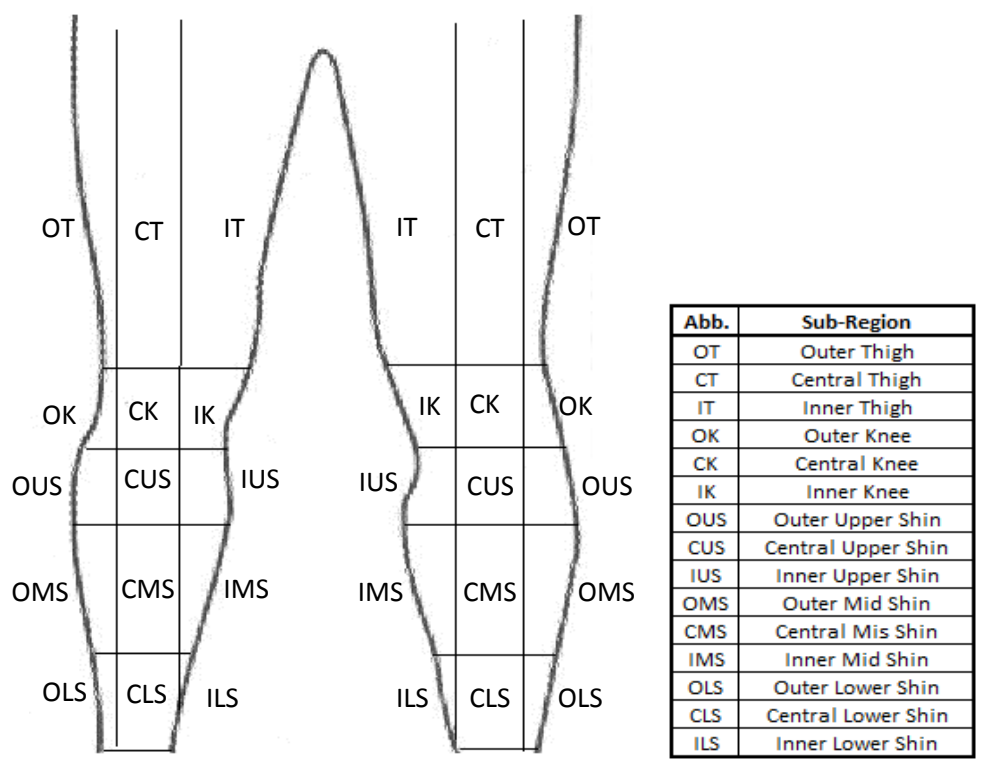

Figure 3. 15 regions of each leg

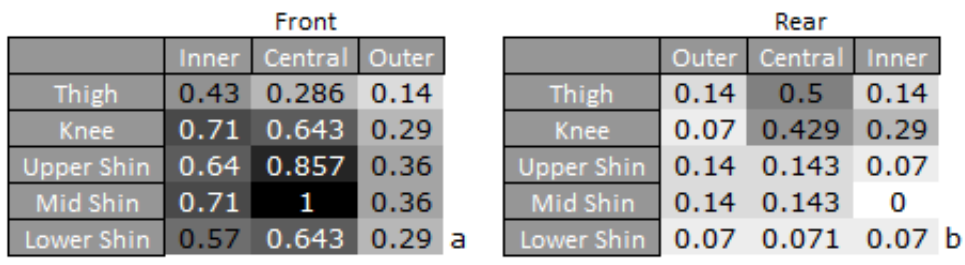

Figure 4. Normalised 'grey-scale' map of the probability of being impacted within certain regions for all 15 shots for a) the front and b) back leg. 

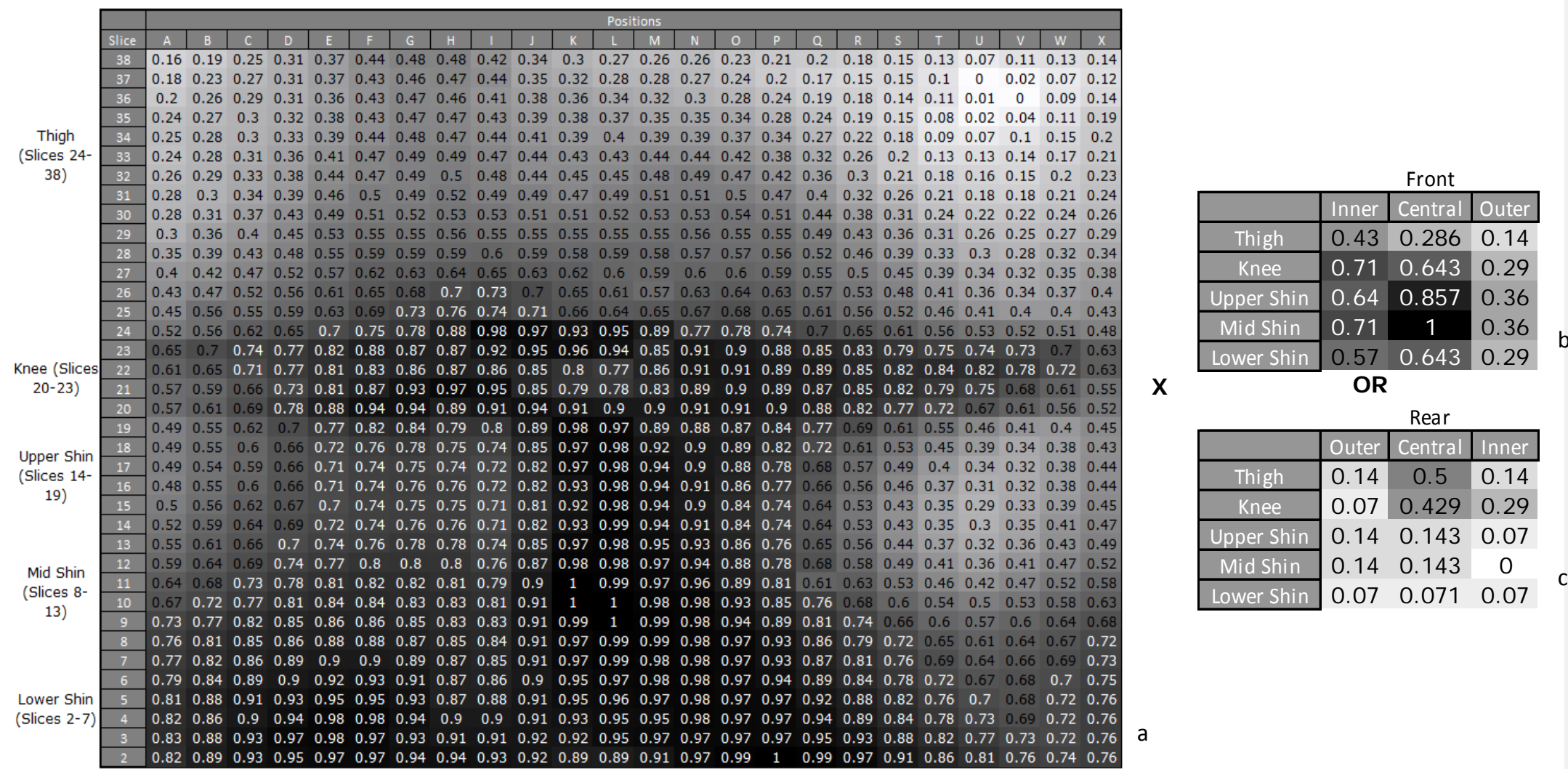

$\mathbf{x}$ OR

\begin{tabular}{|c|c|c|c|}
\hline & \multicolumn{3}{|c|}{ Rear } \\
\hline & Outer & Central & Inner \\
\hline Thigh & 0.14 & 0.5 & 0.14 \\
\hline Knee & 0.07 & 0.429 & 0.29 \\
\hline Upper Shin & 0.14 & 0.143 & 0.07 \\
\hline Mid Shin & 0.14 & 0.143 & 0 \\
\hline Lower Shin & 0.07 & 0.071 & 0.07 \\
\hline
\end{tabular}

Figure 5. a) Normalised potential severity values for a single leg. b) Normalised probability of impact values for front leg of a right-handed batsman. c) Normalised probability value for rear leg of a right-handed batsman. 


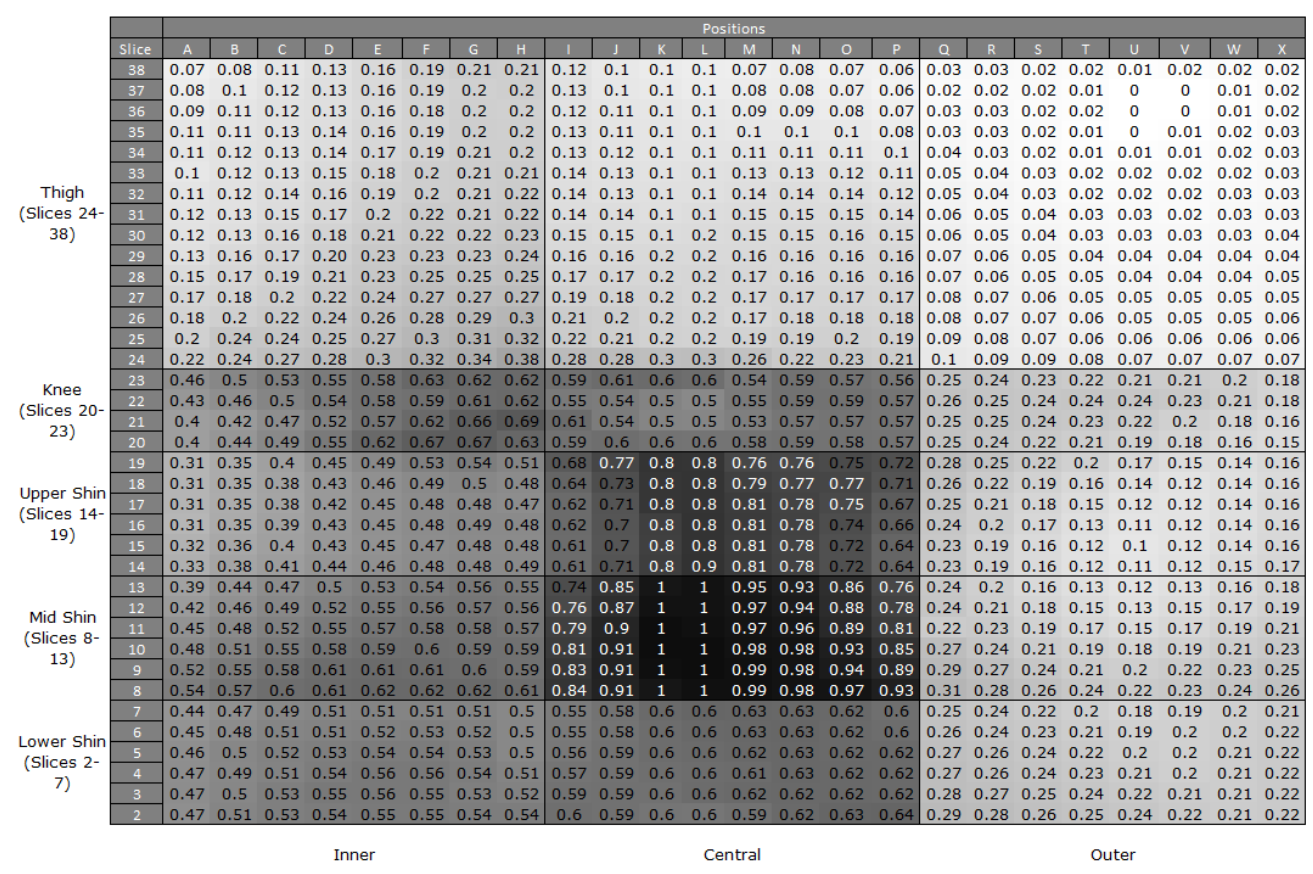

Figure 6. 'Grey-scale' map showing the risk of sustaining an injury in the front leg of a right-handed batsman. 


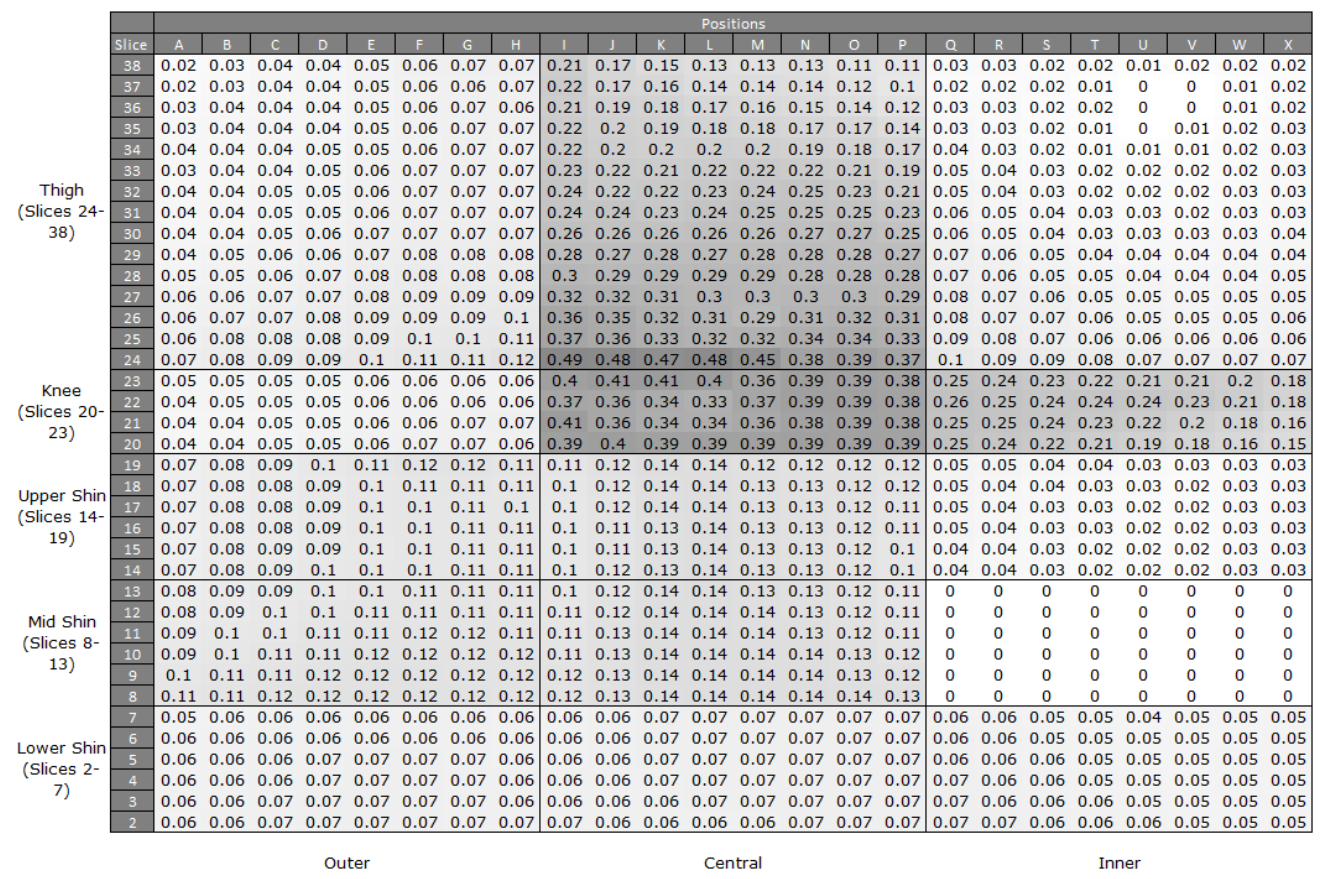

Figure 7. 'Grey-scale' map showing the risk of sustaining an injury in the rear leg of a right-handed batsman. 